\title{
PROJECTIVE NESTED CARTESIAN CODES
}

\author{
CÍCERO CARVALHO, V. G. LOPEZ NEUMANN, AND HIRAM H. LÓPEZ
}

\begin{abstract}
In this paper we introduce a new family of codes, called projective nested cartesian codes. They are obtained by the evaluation of homogeneous polynomials of a fixed degree on a certain subset of $\mathbb{P}^{n}\left(\mathbb{F}_{q}\right)$, and they may be seen as a generalization of the so-called projective Reed-Muller codes. We calculate the length and the dimension of such codes, an upper bound for the minimum distance and the exact minimum distance in a special case (which includes the projective Reed-Muller codes). At the end we show some relations between the parameters of these codes and those of the affine cartesian codes.
\end{abstract}

Keywords: Projective codes; Reed-Muller type codes; Gröbner bases methods.

Mathematics Subject Classification 2010: 14G50; 11T71; 94B27

\section{INTRODUCTION}

Let $K:=\mathbb{F}_{q}$ be a field with $q$ elements and let $A_{0}, \ldots, A_{n}$ be a collection of non-empty subsets of $K$. Consider a projective cartesian set

$$
\mathcal{X}:=\left[A_{0} \times A_{1} \times \cdots \times A_{n}\right]:=\left\{\left(a_{0}: \cdots: a_{n}\right): a_{i} \in A_{i} \text { for all } i\right\} \subset \mathbb{P}^{n},
$$

where $\mathbb{P}^{n}$ is a projective space over the field $K$.

In what follows $d_{i}$ denotes $\left|A_{i}\right|$, the cardinality of $A_{i}$ for $i=0, \ldots, n$. We shall always assume that $2 \leq d_{i} \leq d_{i+1}$ for all $i$.

Let $S:=K\left[X_{0}, \ldots, X_{n}\right]$ be a polynomial ring over the field $K$, let $P_{1}, \ldots, P_{m}$ be the points of $\mathcal{X}$ written with the usual (see e.g. [14, [11, [1) representation for projective points, that is, zeros to the left and the first nonzero entry equal 1 , and let $S_{d}$ be the $K$-vector space of all homogeneous polynomials of $S$ of degree $d$ together with the zero polynomial. The evaluation map

$$
\varphi_{d}: S_{d} \longrightarrow K^{|\mathcal{X}|}, \quad f \mapsto\left(f\left(P_{1}\right), \ldots, f\left(P_{m}\right)\right),
$$

The first and the second authors are partially supported by CNPq and by FAPEMIG. The third author was partially supported by CONACyT and Universidad Autónoma de Aguascalientes

To be published in the Bulletin of the Brazilian Mathematical Society, New Series. The final publication is available at Springer via http://dx.doi.org/doi:10.1007/s00574-016-0010-z . 
defines a linear map of $K$-vector spaces. The image of $\varphi_{d}$, denoted by $C_{\mathcal{X}}(d)$, defines a linear code (as usual by a linear code we mean a linear subspace of $K^{|\mathcal{X}|}$ ). We call $C_{\mathcal{X}}(d)$ a projective cartesian code of order $d$ defined over $A_{0}, \ldots, A_{n}$. Thus the projective cartesian codes are part of the family of evaluation codes defined on a subset of a projective space, see [4], [5], [7] and [10] for other examples. An important special case of the projective cartesian codes, which served as motivation for our work, is the one where $A_{i}=K$ for all $i=0, \ldots, n$. Then we have $\mathcal{X}=\mathbb{P}^{n}$ and $C_{\mathcal{X}}(d)$ is the so-called projective Reed-Muller code (of order $d$ ), as defined and studied in [11] or [14].

The dimension and the length of $C_{\mathcal{X}}(d)$ are given by $\operatorname{dim}_{K} C_{\mathcal{X}}(d)$ (dimension as $K$ vector space) and $|\mathcal{X}|$ respectively. The minimum distance of $C_{\mathcal{X}}(d)$ is given by

$$
\delta_{\mathcal{X}}(d)=\min \left\{\left|\varphi_{d}(f)\right|: \varphi_{d}(f) \neq 0 ; f \in S_{d}\right\}
$$

where $\left|\varphi_{d}(f)\right|$ is the number of non-zero entries of $\varphi_{d}(f)$. These are the main parameters of the code $C_{\mathcal{X}}(d)$ and they are presented in the main results of this paper, although we find the minimum distance only when the $A_{i}^{\prime} s$ satisfy certain conditions (Definition 2.1).

In the next section we compute the length and the dimension of $C_{\mathcal{X}}(d)$, and to do this we use some concepts of commutative algebra which we now recall. The vanishing ideal of $\mathcal{X} \subset \mathbb{P}^{n}$, denoted by $I(\mathcal{X})$, is the ideal of $S$ generated by the homogeneous polynomials that vanish on all points of $\mathcal{X}$. We are interested in the algebraic invariants (degree, Hilbert function) of $I(\mathcal{X})$, because the kernel of the evaluation map, $\varphi_{d}$, is precisely $I(\mathcal{X})_{d}$, where $I(\mathcal{X})_{d}:=S_{d} \cap I(\mathcal{X})$. In general, for any subset (ideal or not) $\mathcal{F}$ of $S$ we define $\mathcal{F}_{d}:=\mathcal{F} \cap S_{d}$. The Hilbert function of $S / I(\mathcal{X})$ is given by

$$
H_{\mathcal{X}}(d):=\operatorname{dim}_{K}\left(S_{d} / I(\mathcal{X})_{d}\right)
$$

so $H_{\mathcal{X}}(d)$ is precisely the dimension of $C_{\mathcal{X}}(d)$.

We will also need tools from Gröbner bases theory, which we recall briefly.

Let $\prec$ be a monomial order defined on the set $\mathcal{M}$ of monomials of the polynomial ring $S$, i.e. $\prec$ is a total order on $\mathcal{M}$, we have $1 \prec M$ for any monomial $M$, and if $M_{1} \prec M_{2}$ then $M M_{1} \prec M M_{2}$ for all $M, M_{1}, M_{2} \in \mathcal{M}$. The largest monomial in a nonzero polynomial $f$ is called the leading monomial of $f$ and is denoted by $\operatorname{lm}(f)$.

Definition 1.1. Let $I$ be an ideal of $S$. A set $\left\{g_{1}, \ldots, g_{s}\right\} \subset I$ is a Gröbner basis for $I$ (with respect to $\prec$ ) if for every $f \in I, f \neq 0$, we have that $\operatorname{lm}(f)$ is a multiple of $\operatorname{lm}\left(g_{i}\right)$ for some $i \in\{1, \ldots, s\}$.

Gröbner basis were introduced in [2] by Bruno Buchberger, who proved that any ideal has a Gröbner basis (with respect to a fixed monomial order). Also it is not difficult to prove 
that if $\left\{g_{1}, \ldots, g_{s}\right\}$ is a Gröbner basis for $I$ then $I=\left\langle g_{1}, \ldots, g_{s}\right\rangle$. An important related concept is that of footprint, which we now present.

Definition 1.2. The footprint (with respect to a monomial order $\prec$ ) of an ideal $I \subset$ $S$, denoted by $\Delta(I)$, is the set of monomials which are not leading monomials of any polynomial in $I$.

The footprint of an ideal $I$ is closely related to a Gröbner basis for $I$ (both being defined with respect to the same monomial order in $\mathcal{M}$ ), as the following result shows.

Proposition 1.3. Let $I \subset S$ be an ideal and let $\left\{g_{1}, \ldots, g_{s}\right\}$ be a Gröbner basis for $I$. Then a monomial $M$ is in $\Delta(I)$ if and only if $M$ is not a multiple of $\operatorname{lm}\left(g_{i}\right)$ for all $i=1, \ldots, s$.

Proof. The "only if" part is obvious from the definition of the footprint. On the other hand, from the definition of Gröbner basis we know that if $M$ is not a multiple of $\operatorname{lm}\left(g_{i}\right)$ for all $i=1, \ldots, s$ then $M$ is not the leading monomial of any polynomial in $I$.

The main property of the footprint, as proved in [2], is that $\{M+I: M \in \Delta(I)\}$ is a basis for $S / I$ as a $K$-vector space. Assume that $I$ is an homogeneous ideal and let $d$ be a nonnegative integer. It is not difficult to see that the classes of the monomials in $\Delta(I)_{d}=\{M \in \Delta(I): \operatorname{deg}(M)=d\}$ form a basis, as a $K$-vector space, for $S_{d} / I_{d}$, which gives a connection between the footprint and the Hilbert function. We can use this to check if a set $G=\left\{g_{1}, g_{2}, \ldots, g_{s}\right\}$ is a Gröbner basis. For this we define $\Delta(G):=$ $\left\{M\right.$ : for all $\left.i, \operatorname{lm}\left(g_{i}\right) \nmid M\right\}$, hence $\Delta(G)_{d}=\{M \in \Delta(G): \operatorname{deg}(M)=d\}$.

Lemma 1.4. Fix a graded monomial order in S. Let $I$ be a homogeneous ideal of $S$ and $G=\left\{g_{1}, g_{2}, \ldots, g_{s}\right\}$ a set of generators of $I$. The set $G$ is a Gröbner Basis of $I$ if and only if the Hilbert function of $I$ is given by $H_{I}(d)=\left|\Delta(G)_{d}\right|$ for all $d \geq 0$.

Proof. If $G$ is a Gröbner basis for $I$ then, from Proposition 1.3 we get that $\Delta(I)=\Delta(G)$, hence $H_{I}(d)=\left|\Delta(G)_{d}\right|$ for all $d \geq 0$. On the other hand, observe that $\Delta(I) \subset \Delta(G)$, and a fortiori $\Delta(I)_{d} \subset \Delta(G)_{d}$ for all $d \geq 0$. If $\left|\Delta(G)_{d}\right|=H_{I}(d)=\left|\Delta(I)_{d}\right|$ then $\Delta(I)=\Delta(G)$ so $G$ is, by definition, a Gröbner basis for $I$.

In the next section the relation between the Hilbert function and the footprint established in the above Lemma will be used to prove that a certain set $\mathcal{G}$ is a Gröbner basis for $\mathcal{X}$ under certain conditions (see Proposition 2.11). Gröbner bases will also play an important role in the proof of Proposition 3.7, which by its turn is a key ingredient in the proof of the main result of Section 3, which determines the minimum distance of a 
particular type of projective cartesian code defined by the product of subfields of $K$ (see Definition (3.4). We will use more than once results about affine cartesian codes, which we now recall.

Let $\mathcal{Y}:=A_{1} \times \cdots \times A_{n} \subset \mathbb{A}^{n}$, where $\mathbb{A}^{n}$ is the $n$-dimensional affine space defined over $K$. For a nonnegative integer $d$ write $S_{\leq d}$ for the $K$-linear subspace of $K^{n}$ formed by the polynomials in $K\left[X_{1}, \ldots, X_{n}\right]$ of degree up to $d$ together with the zero polynomial. Clearly $|\mathcal{Y}|=\Pi_{i=1}^{n} d_{i}=: \tilde{m}$ and let $Q_{1}, \ldots, Q_{\tilde{m}}$ be the points of $\mathcal{Y}$. Define $\phi_{d}: S_{\leq d} \rightarrow K^{\tilde{m}}$ as the evaluation morphism $\left.\phi_{d}(g)=\left(g(Q)_{1}\right), \ldots, g\left(Q_{\tilde{m}}\right)\right)$.

Definition 1.5. The image $C_{\mathcal{Y}}^{*}(d)$ of $\phi_{d}$ is a subvector space of $K^{\tilde{m}}$ called the affine cartesian code (of order $d$ ) defined over the sets $A_{1}, \ldots, A_{n}$.

These codes were introduced in [12], and also appeared independently and in a generalized form in [9]. They are a type of affine variety code, as defined in [8]. In [12] the authors prove that we may ignore sets with just one element, and moreover may always assume that $2 \leq d_{1} \leq \cdots \leq d_{n}$. They also completely determine the parameters of these codes, which are as follows.

Theorem 1.6. [12, Thm. 3.1 and Thm. 3.8]

1) The dimension of $C_{\mathcal{Y}}^{*}(d)$ is $\tilde{m}$ (i.e. $\phi_{d}$ is surjective) if $d \geq \sum_{i=1}^{n}\left(d_{1}-1\right)$, and for $0 \leq d<\sum_{i=1}^{n}\left(d_{1}-1\right)$ we have

$$
\begin{aligned}
\operatorname{dim}\left(C_{\mathcal{Y}}^{*}(d)\right)= & \left(\begin{array}{c}
n+d \\
d
\end{array}\right)-\sum_{i=1}^{n}\left(\begin{array}{c}
n+d-d_{i} \\
d-d_{i}
\end{array}\right) \\
& +\cdots+(-1)^{j} \sum_{1 \leq i_{1}<\cdots<i_{j} \leq n}\left(\begin{array}{c}
n+d-d_{i_{1}}-\cdots-d_{i_{j}} \\
d-d_{i_{1}}-\cdots-d_{i_{j}}
\end{array}\right) \\
& +\cdots+(-1)^{n}\left(\begin{array}{c}
n+d-d_{1}-\cdots-d_{n} \\
d-d_{1}-\cdots-d_{n}
\end{array}\right)
\end{aligned}
$$

where we set $\left(\begin{array}{l}a \\ b\end{array}\right)=0$ if $b<0$.

2) The minimum distance $\delta_{\mathcal{Y}}^{*}(d)$ of $C_{\mathcal{Y}}^{*}(d)$ is 1 , if $d \geq \sum_{i=1}^{n}\left(d_{i}-1\right)$, and for $0 \leq d<$ $\sum_{i=1}^{n}\left(d_{i}-1\right)$ we have

$$
\delta_{\mathcal{Y}}^{*}(d)=\left(d_{k+1}-\ell\right) \prod_{i=k+2}^{n} d_{i}
$$

where $k$ and $\ell$ are uniquely defined by $d=\sum_{i=1}^{k}\left(d_{i}-1\right)+\ell$ with $0 \leq \ell<d_{k+1}-1$ (if $k+1=n$ we understand that $\prod_{i=k+2}^{n} d_{i}=1$, and if $d<d_{1}-1$ then we set $k=0$ and $\ell=d)$. 
We will also use a result from [12] in which the authors determine the (homogeneous) ideal of the set $\overline{\mathcal{Y}}:=\left[1 \times A_{1} \times \cdots \times A_{n}\right]$ (in what follows we use, in a cartesian product, 1 to denote the set $\{1\}$ and 0 to denote the set $\{0\})$.

Theorem 1.7. [12, Thm. 2.5]

$$
I(\overline{\mathcal{Y}})=\left\langle\Pi_{a_{1} \in A_{1}}\left(X_{1}-a_{1} X_{0}\right), \ldots, \Pi_{a_{n} \in A_{n}}\left(X_{n}-a_{n} X_{0}\right)\right\rangle
$$

In [3] there are results on higher Hamming weights of affine cartesian codes, and also a proof of the minimum distance formula stated above which is simpler from the one found in [12 and uses methods similar to the ones used here. We will need a result from [3] which we reproduce here for the reader's convenience.

Lemma 1.8. [3, Lemma 2.1] Let $0<e_{1} \leq \cdots \leq e_{n}$ and $0 \leq s \leq \sum_{i=1}^{n}\left(e_{i}-1\right)$ be integers. Let $m\left(a_{1}, \ldots, a_{n}\right)=\prod_{i=1}^{n}\left(e_{i}-a_{i}\right)$, where $0 \leq a_{i}<e_{i}$ is an integer for all $i=1, \ldots, n$. Then

$$
\min \left\{m\left(a_{1}, \ldots, a_{n}\right): a_{1}+\cdots+a_{n} \leq s\right\}=\left(e_{k+1}-\ell\right) \prod_{i=k+2}^{n} e_{i}
$$

where $k$ and $\ell$ are uniquely defined by $s=\sum_{i=1}^{k}\left(e_{i}-1\right)+\ell$, with $0 \leq \ell<e_{k+1}-1$ (if $s<e_{1}-1$ then take $k=0$ and $\ell=s$, if $k+1=n$ then we understand that $\prod_{i=k+2}^{n} e_{i}=1$ ).

\section{LENGTH AND Dimension}

In this section we define the projective nested cartesian codes and compute their length and dimension. We keep the notation and definitions used in Section 1.

For $A, B$ subsets of $K$ we write $A^{\neq 0}$ to denote the set $A \backslash\{0\}$ and we define $\frac{A}{B}:=$ $\left\{\frac{a}{b}: a \in A, b \in B^{\neq 0}\right\}$.

Definition 2.1. The projective cartesian set $\mathcal{X}=\left[A_{0} \times A_{1} \times \cdots \times A_{n}\right]$ is called projective nested cartesian set if:

(i) for all $i=0, \ldots, n$ we have $0 \in A_{i}$,

(ii) for every $i=1, \ldots, n$ we have $\frac{A_{j}}{A_{i-1}} \subset A_{j}$ for $j=i, \ldots, n$.

For any $d \geq 0$ the associated linear code $C_{\mathcal{X}}(d)$ is called a projective nested cartesian code.

Lemma 2.2. For $A, B$ subsets of $K$ with $0 \in A \cap B$ we have $\frac{A}{B} \subset A \Longleftrightarrow A B \subset A$.

Proof. If $B=\{0\}$ then $\frac{A}{B}=\emptyset, A B=\{0\} \subset A$ and the lemma is true. If $B \supsetneqq\{0\}$ and $\frac{A}{B} \subset A$ then taking $b \in B^{\neq 0}$ we have a bijection $A \rightarrow A$ given by $a \mapsto a / b$ whose inverse 
is the map $a \mapsto a b$, so that $A B \subset A$. Conversely, if $A B \subset A$ taking $b \in B^{\neq 0}$ we have a bijection $A \rightarrow A$ given by $a \mapsto a b$ whose inverse is the map $a \mapsto a / b$, so that $\frac{A}{B} \subset A$.

From the above Lemma we get that condition (ii) of Definition 2.1 is equivalent to the following condition:

(ii') for every $i=1, \ldots, n$ we have $A_{j} A_{i-1} \subset A_{j}$ for $j=i, \ldots, n$.

Example 2.3. If we take $A_{i}=K$ for all $i=0, \ldots, n$ then the the conditions of Definition 2.1 are satisfied, so $\mathbb{P}^{n}$ is a projective nested cartesian set and the projective Reed-Muller codes are projective nested cartesian codes.

Lemma 2.4. If $\mathcal{X}=\left[A_{0} \times A_{1} \times A_{2} \times \cdots \times A_{n}\right]$ is a projective nested cartesian set then

$$
I(\mathcal{X})=\left\langle X_{i} \prod_{a_{j} \in A_{j}}\left(X_{j}-a_{j} X_{i}\right): i<j, i, j=0, \ldots, n\right\rangle .
$$

Proof. We will make an induction on $n$. If $n=1$ then $\mathcal{X}=\left[1 \times A_{n}\right] \cup\{(0: 1)\}$ and from Theorem 1.7 we get $I(\mathcal{X})=\left\langle X_{0} \prod_{a_{1} \in A_{1}}\left(X_{1}-a_{1} X_{0}\right)\right\rangle$. Now we assume that the result is valid for $n-1$. Take $C_{1}:=\left[1 \times A_{1} \times A_{2} \times \cdots \times A_{n}\right], C_{0}:=\left[A_{1} \times A_{2} \times \cdots \times A_{n}\right]$ and $F \in I(\mathcal{X})$. Let $m$ be an element of $C_{0}$ and write

$$
F=F_{1} X_{0}+F_{2}
$$

where $F_{2} \in K\left[X_{1}, \ldots, X_{n}\right]$. As $\mathcal{X}$ is a projective nested cartesian set, $\mathcal{X}=C_{1} \cup\left[0 \times C_{0}\right]$, so $[1, m],[0, m] \in \mathcal{X}$. We have $0=F(0, m)=F_{2}(m)$, then $F_{2} \in I\left(C_{0}\right)$ and by induction

$$
F_{2} \in\left\langle X_{i} \prod_{a_{j} \in A_{j}}\left(X_{j}-a_{j} X_{i}\right): i<j, i, j=1, \ldots, n\right\rangle .
$$

We know also $0=F(1, m)=F_{1}(m)$, then $F_{1} \in I\left(C_{1}\right)$ and from Theorem 1.7 we get

$$
F_{1} \in\left\langle\prod_{a_{i} \in A_{i}}\left(X_{i}-a_{i} X_{0}\right): i=1, \ldots, n\right\rangle .
$$

As $F=F_{1} X_{0}+F_{2}$ the result is true.

Definition 2.5. Let $\mathcal{X}=\left[A_{0} \times \cdots \times A_{n}\right]$ be a projective nested cartesian set. To compute the Hilbert function of $I(\mathcal{X})$ we define

$$
\begin{aligned}
& \mathcal{X}_{i}:=\left[A_{n-i} \times \cdots \times A_{n}\right], \text { so that } I\left(\mathcal{X}_{i}\right) \subset K\left[X_{n-i}, \ldots, X_{n}\right], \text { for } i=0, \ldots, n \text {, and } \\
& \mathcal{X}_{i}^{*}:=\left[1 \times A_{n+1-i} \times \cdots \times A_{n}\right], \text { so that } I\left(\mathcal{X}_{i}^{*}\right) \subset K\left[X_{n-i}, \ldots, X_{n}\right] \text {, for } i=1, \ldots, n .
\end{aligned}
$$

Lemma 2.6. For any positive integer $d, H_{\mathcal{X}_{n}}(d)=H_{\mathcal{X}_{n-1}}(d)+H_{\mathcal{X}_{n}^{*}}(d-1)$. 
Proof. We know that $S_{d}=K\left[X_{1}, \ldots, X_{n}\right]_{d} \bigoplus X_{0} K\left[X_{0}, \ldots, X_{n}\right]_{d-1}$. Let $f \in I\left(\mathcal{X}_{n}\right)_{d}$, then $f=h+X_{0} g$, where $h \in K\left[X_{1}, \ldots, X_{n}\right]_{d}$ and $g \in K\left[X_{0}, \ldots, X_{n}\right]_{d-1}$. By definition 2.5, it is easy to see that $h \in I\left(\mathcal{X}_{n-1}\right)_{d}$ and $g \in I\left(\mathcal{X}_{n}^{*}\right)_{d-1}$ and conversely, if $h \in I\left(\mathcal{X}_{n-1}\right)_{d}$ and $g \in I\left(\mathcal{X}_{n}^{*}\right)_{d-1}$, then $h+X_{0} g \in I\left(\mathcal{X}_{n}\right)_{d}$. Thus $I\left(\mathcal{X}_{n}\right)_{d}=I\left(\mathcal{X}_{n-1}\right)_{d} \bigoplus X_{0} I\left(\mathcal{X}_{n}^{*}\right)_{d-1}$. Then

$$
\begin{aligned}
S_{d} / I\left(\mathcal{X}_{n}\right)_{d} & \simeq K\left[X_{1}, \ldots, X_{n}\right]_{d} / I\left(\mathcal{X}_{n-1}\right)_{d} \oplus X_{0} K\left[X_{0}, \ldots, X_{n}\right]_{d-1} / X_{0} I\left(\mathcal{X}_{n}^{*}\right)_{d-1} \\
& \simeq K\left[X_{1}, \ldots, X_{n}\right]_{d} / I\left(\mathcal{X}_{n-1}\right)_{d} \oplus K\left[X_{0}, \ldots, X_{n}\right]_{d-1} / I\left(\mathcal{X}_{n}^{*}\right)_{d-1}
\end{aligned}
$$

which completes the proof.

Lemma 2.7. Let $\mathcal{X}=\left[A_{0} \times \cdots \times A_{n}\right]$ be a projective nested cartesian set. The Hilbert function of $S / I(\mathcal{X})$ is given by

$$
\begin{aligned}
H_{\mathcal{X}}(d)= & 1+\sum_{j=1}^{n}\left[\left(\begin{array}{c}
j+d-1 \\
d-1
\end{array}\right)-\sum_{i=n+1-j}^{n}\left(\begin{array}{c}
j+d-1-d_{i} \\
d-1-d_{i}
\end{array}\right)\right. \\
& +\cdots+(-1)^{k} \sum_{n+1-j \leq i_{1}<\cdots<i_{k} \leq n}\left(\begin{array}{c}
j+d-1-\left(d_{i_{1}}+\cdots+d_{i_{k}}\right) \\
d-1-\left(d_{i_{1}}+\cdots+d_{i_{k}}\right)
\end{array}\right) \\
& \left.+\cdots+(-1)^{j}\left(\begin{array}{c}
j+d-1-\left(d_{n+1-j}+\cdots+d_{n}\right) \\
d-1-\left(d_{n+1-j}+\cdots+d_{n}\right)
\end{array}\right)\right] .
\end{aligned}
$$

Proof. Using Lemma 2.6 we have $H_{\mathcal{X}}(d)=H_{\mathcal{X}_{0}}(d)+\sum_{j=1}^{n} H_{\mathcal{X}_{j}^{*}}(d-1)$. As $\mathcal{X}_{0}=[1]$, then $I\left(\mathcal{X}_{0}\right)=0$ and $H_{\mathcal{X}_{0}}=1$. From Theorem 1.6 (1) we get

$$
\begin{aligned}
H_{\mathcal{X}_{j}^{*}}(d-1)= & \left(\begin{array}{c}
j+d-1 \\
d-1
\end{array}\right)-\sum_{i=n+1-j}^{n}\left(\begin{array}{c}
j+d-1-d_{i} \\
d-1-d_{i}
\end{array}\right) \\
& +\cdots+(-1)^{k} \sum_{n+1-j \leq i_{1}<\cdots<i_{k} \leq n}\left(\begin{array}{c}
j+d-1-\left(d_{i_{1}}+\cdots+d_{i_{k}}\right) \\
d-1-\left(d_{i_{1}}+\cdots+d_{i_{k}}\right)
\end{array}\right) \\
& +\cdots+(-1)^{j}\left(\begin{array}{c}
j+d-1-\left(d_{n+1-j}+\cdots+d_{n}\right) \\
d-1-\left(d_{n+1-j}+\cdots+d_{n}\right)
\end{array}\right) .
\end{aligned}
$$

We come to the main result of this section. 
8

CÍCERO CARVALHO, V. G. LOPEZ NEUMANN, AND HIRAM H. LÓPEZ

Theorem 2.8. Let $C_{\mathcal{X}}(d)$ be a projective nested cartesian code over $A_{0}, \ldots, A_{n}$. The length of the code is given by $m=1+\sum_{i=1}^{n} d_{i} \cdots d_{n}$ and its dimension by

$$
\begin{aligned}
\operatorname{dim}_{K} C_{\mathcal{X}}(d)= & 1+\sum_{j=1}^{n}\left[\left(\begin{array}{c}
j+d-1 \\
d-1
\end{array}\right)-\sum_{i=n+1-j}^{n}\left(\begin{array}{c}
j+d-1-d_{i} \\
d-1-d_{i}
\end{array}\right)\right. \\
& +\cdots+(-1)^{k} \sum_{n+1-j \leq i_{1}<\cdots<i_{k} \leq n}\left(\begin{array}{c}
j+d-1-\left(d_{i_{1}}+\cdots+d_{i_{k}}\right) \\
d-1-\left(d_{i_{1}}+\cdots+d_{i_{k}}\right)
\end{array}\right) \\
& \left.+\cdots+(-1)^{j}\left(\begin{array}{c}
j+d-1-\left(d_{n+1-j}+\cdots+d_{n}\right) \\
d-1-\left(d_{n+1-j}+\cdots+d_{n}\right)
\end{array}\right)\right] .
\end{aligned}
$$

Proof. As $\mathcal{X}=\left[A_{0} \times A_{1} \times \cdots \times A_{n}\right]$ is a projective nested cartesian set, then

$$
\begin{gathered}
\mathcal{X}=\left[A_{0}^{\neq 0} \times A_{1} \times A_{2} \times \cdots \times A_{n}\right] \\
\cup\left[0 \times A_{1}^{\neq 0} \times A_{2} \times \cdots \times A_{n}\right] \\
\vdots \\
\cup\left[0 \times 0 \times 0 \times \cdots \times A_{n-1}^{\neq 0} \times A_{n}\right] \\
\cup[0 \times 0 \times 0 \times \cdots \times 0 \times 1] .
\end{gathered}
$$

Condition (ii) of Definition 2.1 allows us change $A_{i}^{\neq 0}$ for 1 for all $i=0, \ldots, n-1$ so we get $|\mathcal{X}|=1+\sum_{i=1}^{n} d_{i} \cdots d_{n}$. As the kernel of the evaluation map $\varphi_{d}$ is $S_{d} \cap I(\mathcal{X})$, the Hilbert function of $S / I(\mathcal{X})$ agrees with the dimension of $C_{\mathcal{X}}(d)$, so, by Lemma 2.7 we have the dimension.

From now on we choose the graded lexicographic monomial order $\prec$ in $S$, where $X_{0} \prec$ $\cdots \prec X_{n}$, and to finish this section we show that the set

$$
\mathcal{G}:=\left\{X_{i} \prod_{a_{j} \in A_{j}}\left(X_{j}-a_{j} X_{i}\right): i<j, \quad i, j=0, \ldots, n\right\}
$$

is a Gröbner basis of the ideal $I(\mathcal{X})$. In what follows $M$ denotes a monomial in $S$. 
Lemma 2.9. The number of elements of $\Delta(\mathcal{G})_{d}$ is given by

$$
\begin{aligned}
& \left(\begin{array}{c}
n+d \\
n
\end{array}\right)-\sum_{j=1}^{n}\left(\left(\begin{array}{c}
n+d-d_{j} \\
n
\end{array}\right)-\left(\begin{array}{c}
n-j+d-d_{j} \\
n-j
\end{array}\right)\right) \\
& +\cdots+(-1)^{k} \sum_{1 \leq j_{1}<\cdots<j_{k} \leq n}\left(\left(\begin{array}{c}
n+d-\left(d_{j_{1}}+\cdots+d_{j_{k}}\right) \\
n
\end{array}\right)-\left(\begin{array}{c}
n-j_{1}+d-\left(d_{j_{1}}+\cdots+d_{j_{k}}\right) \\
n-j_{1}
\end{array}\right)\right) \\
& +\cdots+(-1)^{n}\left(\begin{array}{c}
n+d-\left(d_{1}+\cdots+d_{n}+1\right) \\
n
\end{array}\right) .
\end{aligned}
$$

Proof. Observe that $\Delta(\mathcal{G})=\left\{M: X_{i} X_{j}^{d_{j}} \nmid M, 0 \leq i<j \leq n\right\}$. For $1 \leq j \leq n$, we define $\mathcal{M}_{j}:=\left\{M:\right.$ there is $\left.i, 0 \leq i<j, X_{i} X_{j}^{d_{j}} \mid M\right\}$. Then $\Delta(\mathcal{G})=\mathcal{M}_{S}-\left(\bigcup_{j=1}^{n} \mathcal{M}_{j}\right)$, where $\mathcal{M}_{S}$ is the set of all monomials in $S$. Therefore, when we count the number of monomials of degree $d$ in $\Delta(\mathcal{G})$, from the inclusion-exclusion theorem we get

$$
\begin{aligned}
\Delta(\mathcal{G})_{d}= & \left|\left(\mathcal{M}_{S}\right)_{d}\right|-\sum_{j=1}^{n}\left|\left(\mathcal{M}_{j}\right)_{d}\right|+\sum_{j_{1}<j_{2}}\left|\left(\mathcal{M}_{j_{1}} \cap \mathcal{M}_{j_{2}}\right)_{d}\right| \\
& -\cdots+(-1)^{k} \sum_{j_{1}<j_{2}<\cdots<j_{k}}\left|\left(\mathcal{M}_{j_{1}} \cap \mathcal{M}_{j_{2}} \cap \cdots \cap \mathcal{M}_{j_{k}}\right)_{d}\right| \\
& +\cdots+(-1)^{n}\left|\left(\mathcal{M}_{1} \cap \mathcal{M}_{2} \cap \cdots \cap \mathcal{M}_{n}\right)_{d}\right|
\end{aligned}
$$

Clearly $\left|\left(\mathcal{M}_{S}\right)_{d}\right|=\left(\begin{array}{c}n+d \\ n\end{array}\right)$. Let $j \in\{1, \ldots, n\}$ and let $M=X_{0}^{\alpha_{0}} \cdots . X_{n}^{\alpha_{n}} \in\left(\mathcal{M}_{j}\right)_{d}$, then there exists $i<j$, such that $\alpha_{i} \geq 1$ and $\alpha_{j} \geq d_{j}$. Taking $\beta_{j}=\alpha_{j}-d_{j}$ and for $k \neq j$, $\beta_{k}=\alpha_{k}$, we have that $\left|\left(\mathcal{M}_{j}\right)_{d}\right|$ is the number of solutions of $\beta_{0}+\cdots+\beta_{n}=d-d_{j}$, such that $\beta_{0}+\cdots+\beta_{j-1} \geq 1$. Then $\left|\left(\mathcal{M}_{j}\right)_{d}\right|$ is the number of solutions of $\beta_{0}+\cdots+\beta_{n}=d-d_{j}$ minus the number of solutions of $\beta_{j}+\cdots+\beta_{n}=d-d_{j}$. This means

$$
\left|\left(\mathcal{M}_{j}\right)_{d}\right|=\left(\begin{array}{c}
n+d-d_{j} \\
n
\end{array}\right)-\left(\begin{array}{c}
n-j+d-d_{j} \\
n-j
\end{array}\right)
$$

Now let $M=X_{0}^{\alpha_{0}} \cdots \cdot X_{n}^{\alpha_{n}} \in\left(\mathcal{M}_{j_{1}} \cap \cdots \cap \mathcal{M}_{j_{k}}\right)_{d}$, then there exists $i<j_{1}$, such that $\alpha_{i} \geq 1$ and $\alpha_{j_{w}} \geq d_{j_{w}}$, for $1 \leq w \leq k$. Taking $\beta_{j_{w}}=\alpha_{j_{w}}-d_{j_{w}}$, for $1 \leq w \leq k$, with $l \neq j_{w}$ and $\beta_{l}=\alpha_{l}$, we get that $\left|\left(\mathcal{M}_{j_{1}} \cap \cdots \cap \mathcal{M}_{j_{k}}\right)_{d}\right|$ is the number of solutions of $\beta_{0}+\cdots+\beta_{n}=$ $d-\left(d_{j_{1}}+\cdots+d_{j_{k}}\right)$ minus the number of solutions of $\beta_{j_{1}}+\cdots+\beta_{n}=d-\left(d_{j_{1}}+\cdots+d_{j_{k}}\right)$, hence

$$
\left|\left(\mathcal{M}_{j_{1}} \cap \cdots \cap \mathcal{M}_{j_{k}}\right)_{d}\right|=\left(\begin{array}{c}
n+d-\left(d_{j_{1}}+\cdots+d_{j_{k}}\right) \\
n
\end{array}\right)-\left(\begin{array}{c}
n-j_{1}+d-\left(d_{j_{1}}+\cdots+d_{j_{k}}\right) \\
n-j_{1}
\end{array}\right) .
$$


For $k=n$ we have

$$
\begin{aligned}
\left(\begin{array}{c}
n+d-\left(d_{1}+\cdots+d_{n}\right) \\
n
\end{array}\right) & -\left(\begin{array}{c}
n-1+d-\left(d_{1}+\cdots+d_{n}\right) \\
n-1
\end{array}\right) \\
& =\left(\begin{array}{c}
n+d-\left(d_{1}+\cdots+d_{n}+1\right) \\
n
\end{array}\right) .
\end{aligned}
$$

We use the next well-known combinatorial result to check that $H_{\mathcal{X}}(d)=\left|\Delta(\mathcal{G})_{d}\right|$ for all $d \geq 0$.

Lemma 2.10. Let $a, b$ be non-negative integers. Then $\sum_{j=0}^{a}\left(\begin{array}{c}j+b-1 \\ j\end{array}\right)=\left(\begin{array}{c}a+b \\ a\end{array}\right)$.

Proposition 2.11. Let $\mathcal{X}=\left[A_{0} \times \cdots \times A_{n}\right]$ be a projective nested cartesian set. The set $\mathcal{G}=\left\{X_{i} \prod_{a_{j} \in A_{j}}\left(X_{j}-a_{j} X_{i}\right): i<j, i, j=0, \ldots, n\right\}$ is a Gröbner basis for $I(\mathcal{X})$.

Proof. From Lemma 1.4 we only need to compare the formulas of Lemmas 2.7 and 2.9. On the formula for the Hilbert Function, we distribute the sum, use Lemma 2.10 and compare term by term with the formula for the footprint. The first term is

$$
1+\sum_{j=1}^{n}\left(\begin{array}{c}
j+d-1 \\
d-1
\end{array}\right)=\sum_{j=0}^{n}\left(\begin{array}{c}
j+d-1 \\
j
\end{array}\right)=\left(\begin{array}{c}
n+d \\
n
\end{array}\right)
$$

the second term is

$$
\begin{aligned}
\sum_{j=1}^{n} \sum_{i=n+1-j}^{n}\left(\begin{array}{c}
j+d-1-d_{i} \\
d-1-d_{i}
\end{array}\right) & =\sum_{i=1}^{n} \sum_{j=n+1-i}^{n}\left(\begin{array}{c}
j+d-1-d_{i} \\
j
\end{array}\right) \\
& =\sum_{j=1}^{n} \sum_{i=n+1-j}^{n}\left(\begin{array}{c}
i+d-1-d_{j} \\
i
\end{array}\right) \\
& =\sum_{j=1}^{n}\left(\sum_{i=0}^{n}\left(\begin{array}{c}
i+d-d_{j}-1 \\
i
\end{array}\right)-\sum_{i=0}^{n-j}\left(\begin{array}{c}
i+d-d_{j}-1 \\
i
\end{array}\right)\right) \\
& =\sum_{j=1}^{n}\left(\left(\begin{array}{c}
n+d-d_{j} \\
n
\end{array}\right)-\left(\begin{array}{c}
n-j+d-d_{j} \\
n-j
\end{array}\right)\right),
\end{aligned}
$$


and the general term is

$$
\begin{aligned}
& \sum_{j=1}^{n} \sum_{n+1-j \leq i_{1}<\cdots<i_{k} \leq n}\left(\begin{array}{c}
j+d-1-\left(d_{i_{1}}+\cdots+d_{i_{k}}\right) \\
d-1-\left(d_{i_{1}}+\cdots+d_{i_{k}}\right)
\end{array}\right) \\
& =\sum_{1 \leq i_{1}<\cdots<i_{k} \leq n} \sum_{j=n+1-i_{1}}^{n}\left(\begin{array}{c}
j+d-1-\left(d_{i_{1}}+\cdots+d_{i_{k}}\right) \\
j
\end{array}\right) \\
& =\sum_{1 \leq i_{1}<\cdots<i_{k} \leq n}\left(\left(\begin{array}{c}
n+d-\left(d_{i_{1}}+\cdots+d_{i_{k}}\right) \\
n
\end{array}\right)-\left(\begin{array}{c}
n-i_{1}+d-\left(d_{i_{1}}+\cdots+d_{i_{k}}\right) \\
n-i_{1}
\end{array}\right)\right) .
\end{aligned}
$$

Finally, for the last term, the sum on the formula for the Hilbert function has only one term, and

$$
\left(\begin{array}{c}
n+d-1-\left(d_{1}+\cdots+d_{n}\right) \\
d-1-\left(d_{1}+\cdots+d_{n}\right)
\end{array}\right)=\left(\begin{array}{c}
n+d-\left(d_{1}+\cdots+d_{n}+1\right) \\
n
\end{array}\right)
$$

which proves the Proposition.

\section{Minimum Distance}

We start this section by presenting an upper bound for the minimum distance of projective nested cartesian codes. Instead of $f\left(X_{0}, \ldots, X_{n}\right)$ we write simply $f(X)$ for a polynomial in $S$.

Lemma 3.1. If $\mathcal{X}$ is the projective nested cartesian set over $A_{0}, \ldots, A_{n}$, then the minimum distance of $C_{\mathcal{X}}(d)$ satisfies $\delta_{\mathcal{X}}(d) \leq\left(d_{k+1}-\ell\right) d_{k+2} \cdots d_{n}$ if $1 \leq d \leq \sum_{i=1}^{n}\left(d_{i}-1\right)$, and $\delta_{\mathcal{X}}(d)=1$ in otherwise, where $0 \leq k \leq n-1$ and $0 \leq \ell<d_{k+1}-1$ are the unique integers such that $d-1=\sum_{i=1}^{k}\left(d_{i}-1\right)+\ell$.

Proof. For all $i=0, \ldots, n$ choose $a_{i} \in A_{i}$. It is easy to see that the polynomial

$$
f(X)=X_{0} \prod_{i=1}^{n} \prod_{a \in A_{i}}^{a \neq a_{i}}\left(X_{i}-a X_{0}\right)
$$

of degree $\sum_{i=1}^{n}\left(d_{i}-1\right)+1$ is zero for all points of $\mathcal{X}$ except $\left(1: a_{1}: \cdots: a_{n}\right)$. Thus for $d>\sum_{i=1}^{n}\left(d_{i}-1\right)$ we get $\delta_{\mathcal{X}}(d)=1$. Let $B_{k+1} \subset A_{k+1}$ be a set with $\ell$ elements. For 
$d-1=\sum_{i=1}^{k}\left(d_{i}-1\right)+\ell$, taking

$$
f(X)=X_{0}\left(\prod_{i=1}^{k} \prod_{a \in A_{i}}^{a \neq a_{i}}\left(X_{i}-a X_{0}\right)\right)\left(\prod_{a \in B_{k+1}}\left(X_{k+1}-a X_{0}\right)\right)
$$

we obtain the desired inequality.

The next example, found by R. Villarreal, proves that this upper bound is not reached in all cases, but we will prove that it is the true value of the minimum distance in some special cases, which include the projective Reed-Muller codes (see Theorem 3.8).

Example 3.2. Let $K=\mathbb{F}_{4}$ be a finite field with 4 elements and let $K_{0}=K_{1}=\mathbb{F}_{2}, K_{2}=$ $\mathbb{F}_{4}$ be subsets of $K$. Then $\mathcal{X}=\left[K_{0} \times K_{1} \times K_{2}\right]$ is a projective nested cartesian product, and the minimum distance of the code $C_{\mathcal{X}}(d)$ is:

$$
\begin{array}{c|l|l|l|l}
d & 1 & 2 & 3 & 4 \\
\hline \delta_{\mathcal{X}}(d) & 8 & 4 & 3 & 1
\end{array}
$$

Observe that for $d=4$, we have $d-1=(2-1)+2$ and for $f=X_{2}\left(X_{2}^{3}+X_{1}^{3}+X_{0}^{3}+X_{0}^{2} X_{1}\right)$, we have $w(f)=1<\left(d_{k+1}-\ell\right)=(4-2)=2$.

Lemma 3.3. Let $\mathcal{X}=\left[A_{0} \times \cdots \times A_{n}\right]$ be a projective nested cartesian set. For all $j=$ $0, \ldots, n$ let $a_{j} \in A_{j}^{\neq 0}$ and define $B_{j}=a_{j}^{-1} A_{j}$. Then $\mathcal{Y}=\left[B_{0} \times \cdots \times B_{n}\right]$ is a projective nested cartesian set such that $1 \in B_{j}$, for all $j=0, \ldots, n$, and $C_{\mathcal{X}}(d)=C_{\mathcal{Y}}(d)$, for all degree $d$.

Proof. Let $\mathcal{X}=\left\{P_{1}, \ldots, P_{m}\right\}$ and $\mathcal{Y}=\left\{Q_{1}, \ldots, Q_{m}\right\}$, where $P_{i}=\left(x_{0}: \cdots: x_{n}\right)$ and $Q_{i}=\left(a_{0}^{-1} x_{0}, \ldots, a_{n}^{-1} x_{n}\right)$ for all $i=0, \ldots, n$. Let $v \in C_{\mathcal{X}}(d)$, then $v=\left(f\left(P_{1}\right): \cdots: f\left(P_{m}\right)\right)$ for some $f \in S_{d}$. Define $g\left(X_{0}, \ldots, X_{n}\right)=f\left(a_{0} X_{0}, \ldots, a_{n} X_{n}\right) \in S_{d}$. It is easy to see that $v=\left(g\left(Q_{1}\right): \cdots: g\left(Q_{m}\right)\right)$, so that $C_{\mathcal{X}}(d) \subset C_{\mathcal{Y}}(d)$. The proof of $C_{\mathcal{Y}}(d) \subset C_{\mathcal{X}}(d)$ is similar.

Thus we see that one may always assume that $1 \in A_{j}$, for all $j=0, \ldots, n$. We present now the special class of projective nested cartesian set for whose associated codes we will determine the minimum distance.

Definition 3.4. Let $K_{0} \subset \cdots \subset K_{n}$ be subfields of $K$, with $\left|K_{i}\right|=d_{i}$ for all $0 \leq i \leq n$. Observe that $d_{i+1}=d_{i}^{r_{i}}$, for some $r_{i} \geq 1$ and $q=d_{n}^{r_{n}}$. Then $\mathcal{X}=\left[K_{0} \times \cdots \times K_{n}\right]$ is a projective nested cartesian set which is called a projective nested product of fields. 
Clearly $\mathbb{P}^{n}$ is a projective nested product of fields, so our results on codes defined over such sets extend the results on projective Reed-Muller codes.

Definition 3.5. For a set $\mathcal{A} \subset \mathcal{X}$ and $f \in S_{d} \backslash I(\mathcal{A})$, define

$$
Z_{\mathcal{A}}(f):=\{P \in \mathcal{A}: f(P)=0\} .
$$

In this way, for a codeword $v=\left(f\left(P_{1}\right), \ldots, f\left(P_{m}\right)\right) \neq 0$, where $f(X) \in S_{d} \backslash I(\mathcal{X})_{d}$, the weight of $v$ is $\left|\mathcal{X} \backslash Z_{\mathcal{X}}(f)\right|$, and the minimum distance of $C_{\mathcal{X}}(d)$ is

$$
\delta_{\mathcal{X}}(d)=\min \left\{\left|\mathcal{X} \backslash Z_{\mathcal{X}}(f)\right|: f \in S_{d} \backslash I(\mathcal{X})_{d}\right\} .
$$

Lemma 3.6. Let $f$ be an element of $S_{d}$ such that for all $t \leq j \leq n$ we have $Z_{\mathcal{X}}\left(X_{j}\right) \subset$ $Z_{\mathcal{X}}(f)$. Then there exists $g_{t}(X)$ in $S_{d-(n-t+1)}$ such that $f-g_{t} \cdot X_{t} \cdots X_{n} \in I(\mathcal{X})$.

Proof. Write $f=g_{n} X_{n}+h_{n}$, where $h_{n} \in K\left[X_{0}, \ldots, X_{n-1}\right]_{d}$. For any $P=\left(x_{0}: \cdots\right.$ : $\left.x_{n-1}: 0\right) \in \mathcal{X}$, we have $f(P)=0$. This implies that $h_{n} \in I\left(\left[K_{0} \times \cdots \times K_{n-1}\right]\right)$, and a fortiori we have $h_{n} \in I(\mathcal{X})$. By induction on $\alpha$, suppose that for some $t+1 \leq \alpha \leq n$ we have $f=g_{\alpha} X_{\alpha} \cdots X_{n}+h_{\alpha}$, where $h_{\alpha} \in I(\mathcal{X})$. Write $g_{\alpha}=g_{\alpha-1} X_{\alpha-1}+\tilde{h}_{\alpha-1}$, where $\tilde{h}_{\alpha-1} \in K\left[X_{0}, \ldots, X_{\alpha-2}, X_{\alpha} \ldots, X_{n}\right]$. For any $P=\left(x_{0}: \cdots: x_{\alpha-2}: 0: x_{\alpha}: \cdots: x_{n}\right) \in \mathcal{X}$, we have $f(P)=0$. This implies $\left(\tilde{h}_{\alpha-1} X_{\alpha} \cdots X_{n}\right)(P)=0$, which means $\tilde{h}_{\alpha-1} X_{\alpha} \cdots X_{n} \in$ $I\left(\left[K_{0} \times \cdots \times K_{\alpha-2} \times K_{\alpha} \times \cdots \times K_{n}\right]\right) \subset I(\mathcal{X})$. We have then

$$
f=g_{\alpha-1} X_{\alpha-1} \cdots X_{n}+\tilde{h}_{\alpha-1} X_{\alpha} \cdots X_{n}+h_{\alpha},
$$

where $\tilde{h}_{\alpha-1} X_{\alpha} \cdots X_{n}+h_{\alpha} \in I(\mathcal{X})$. By induction on $\alpha$, our result is proved. It is easy to see that $g_{t} \in S_{d-(n-t+1)}$.

Proposition 3.7. Let $\mathcal{X}$ be the projective nested product of fields over $K_{0}, \ldots, K_{n}$, and let $f \in S_{d} \backslash I(\mathcal{X})$ be a homogeneous polynomial on $S$ of degree $d$, with $1 \leq d \leq \sum_{i=1}^{n}\left(d_{i}-1\right)$ if $d_{1}=\cdots=d_{n}$, or $1 \leq d<d_{r+1}$ if there exists a positive integer $r$ such that $d_{1}=d_{r}<d_{r+1}$. Then

$$
\left|\mathcal{X} \backslash Z_{\mathcal{X}}(f)\right| \geq\left(d_{k+1}-\ell\right) d_{k+2} \cdots d_{n}
$$

where $0 \leq k \leq n-1$ and $0 \leq \ell<d_{k+1}-1$ are the unique integers such that $d-1=$ $\sum_{i=1}^{k}\left(d_{i}-1\right)+\ell$

Proof. We will make an induction on $n$. Let $n=1$ and $f \in S_{d} \backslash I(\mathcal{X})$, where $1 \leq d \leq d_{1}-1$. Then $f$ has no more than $d$ roots in $\mathbb{P}^{1}$ and a fortiori in $\mathcal{X}=\left[K_{0} \times K_{1}\right]$. Thus anyway we have

$$
\left|\mathcal{X} \backslash Z_{\mathcal{X}}(f)\right| \geq\left(d_{1}+1\right)-d=d_{1}-(d-1) .
$$


So now we assume that the statement of the theorem holds for the product $\left[K_{0} \times K_{1} \times\right.$ $\left.\cdots \times K_{n-1}\right]$. Define

$$
\mathcal{Y}_{n}^{*}=\left[1 \times K_{1} \times \cdots \times K_{n}\right] \text { and } \mathcal{Y}_{n-1}=\left[0 \times K_{1} \times \cdots \times K_{n}\right]
$$

in particular $\mathcal{X}=\mathcal{Y}_{n}^{*} \cup \mathcal{Y}_{n-1}$. Let $f \in S_{d} \backslash I(\mathcal{X})$ be a polynomial of degree $d$, with $d$ as in the statement of the proposition.

Suppose firstly that $f \in I\left(\mathcal{Y}_{n}^{*}\right)$ (so $f \notin I\left(\mathcal{Y}_{n-1}\right)$ ). From Theorem 1.7 (and the fact that $K_{j}$ is a finite field with $d_{j}$ elements, for $\left.j=1, \ldots, n\right)$ we get that $I\left(\mathcal{Y}_{n}^{*}\right)$ is generated by $\tilde{\mathcal{G}}=\left\{X_{j}^{d_{j}}-X_{j} X_{0}^{d_{j}-1} \mid j=1, \ldots, n\right\}$. Endowing $S$ with a graded-lexicographic order $\prec$ such that $X_{0} \prec X_{1} \prec \cdots \prec X_{n}$ we get that $\operatorname{lm}\left(X_{j}^{d_{j}}-X_{j} X_{0}^{d_{j}-1}\right)=X_{j}^{d_{j}}$, for all $j=1, \ldots, n$. Thus any pair of these leading monomials are coprime, so $\tilde{\mathcal{G}}$ is a Gröbner basis for $I\left(\mathcal{Y}_{n}^{*}\right)$, with respect to $\prec$ (see [6, p. 104]). Dividing $f$ by the elements of $\tilde{\mathcal{G}}$ we find homogeneous polynomials $g_{j}$ such that $f(X)=\sum_{j=1}^{n} g_{j}(X)\left(X_{j}^{d_{j}}-X_{j} X_{0}^{d_{j}-1}\right)$. Observe that if $d_{1}=\cdots=d_{n}$ then $g_{j}$, if nonzero, has degree $d-d_{j}(j=1, \ldots, n)$. On the other hand, if $d_{1}=d_{r}<d_{r+1}$, then from $d<d_{r+1}$ we have $g_{j}=0$ for $j>r$, and for $j \in\{1, \ldots, r$ we have that $g_{j}$, if nonzero, is of has degree $d-d_{j}$. Define $g(X):=\sum_{j=1}^{n} g_{j}(X) X_{j}$, which is a homogeneous polynomial of degree $\tilde{d}=d-d_{1}+1$. Observe that $\left.g\right|_{\mathcal{Y}_{n-1}}=\left.f\right|_{\mathcal{Y}_{n-1}}$ and since $f \notin I\left(\mathcal{Y}_{n-1}\right)$, we must have $g \notin I\left(\mathcal{Y}_{n-1}\right)$, and as $\tilde{d}-1=d-1-\left(d_{1}-1\right)=\sum_{i=2}^{k}\left(d_{i}-1\right)+\ell$, we can apply the induction hypothesis obtaining

$$
\left|\mathcal{X} \backslash Z_{\mathcal{X}}(f)\right|=\left|\mathcal{Y}_{n-1} \backslash Z_{\mathcal{Y}_{n-1}}(g)\right| \geq\left(d_{k+1}-\ell\right) d_{k+2} \cdots d_{n}
$$

This proves the proposition in the case where $f \in I\left(\mathcal{Y}_{n}^{*}\right)$ and $f \notin I\left(\mathcal{Y}_{n-1}\right)$.

Suppose now that $f \in I\left(\mathcal{Y}_{n-1}\right)$ and $f \notin I\left(\mathcal{Y}_{n}^{*}\right)$, and write $f=h+X_{0} g$ where $h(X)=$ $f\left(0, X_{1}, \ldots, X_{n}\right)$. Since $\left.f\right|_{\mathcal{Y}_{n-1}}=0$ we have $\left.h\right|_{\mathcal{Y}_{n-1}}=0$ and a fortiori $\left.h\right|_{\mathcal{Y}_{n}^{*}}=0$ so $h \in I(\mathcal{X})$. Observe that $\left.f\right|_{\mathcal{Y}_{n}^{*}}=\left.g\right|_{\mathcal{Y}_{n}^{*}}$ and clearly the number of zeros of $g$ in $\mathcal{Y}_{n}^{*}$ is the same of the number of zeros of $g\left(1, X_{1}, \ldots, X_{n}\right)$ in the cartesian product $K_{1} \times \cdots \times K_{n}$. Since $\operatorname{deg}\left(g\left(1, X_{1}, \ldots, X_{n}\right)\right) \leq d-1$ a lower bound for the number of nonzeros of $g$ in $\mathcal{Y}_{n}^{*}$ may be obtained from Theorem 1.6, and we have

$$
\left|\mathcal{X} \backslash Z_{\mathcal{X}}(f)\right|=\left|\mathcal{Y}_{n}^{*} \backslash Z_{\mathcal{Y}_{n}^{*}}(g)\right| \geq\left(d_{k+1}-\ell\right) d_{k+2} \cdots d_{n}
$$

Finally suppose that $f \notin I\left(\mathcal{Y}_{n}^{*}\right)$ and $f \notin I\left(\mathcal{Y}_{n-1}\right)$. 
For $k=n-1$, i.e. when $d=\sum_{i=1}^{n-1}\left(d_{i}-1\right)+\ell+1$, we have

$$
\left|\mathcal{Y}_{n}^{*} \backslash Z_{\mathcal{Y}_{n}^{*}}(f)\right| \geq d_{n}-\ell-1
$$

since, as above, we may consider the number of nonzero points of $f\left(1, X_{1}, \ldots, X_{n}\right)$ in $K_{1} \times \cdots \times K_{n}$ and use Theorem [1.6. From $f \notin I\left(\mathcal{Y}_{n-1}\right)$ we get

$$
\left|\mathcal{Y}_{n-1} \backslash Z_{\mathcal{Y}_{n-1}}(f)\right| \geq 1
$$

which implies

$$
\left|\mathcal{X} \backslash Z_{\mathcal{X}}(f)\right| \geq d_{n}-\ell
$$

and settles the case $k=n-1$. We treat now the case $k<n-1$, and we start by assuming that $\ell+d_{1} \leq d_{k+1}$.

We have that $d=\sum_{i=1}^{k}\left(d_{i}-1\right)+\ell+1$ and $d-1=\sum_{i=2}^{k}\left(d_{i}-1\right)+\ell+d_{1}-1$, then from Theorem 1.6 (2) we get that

$$
\left|\mathcal{Y}_{n}^{*} \backslash Z_{\mathcal{Y}_{n}^{*}}(f)\right| \geq\left(d_{k+1}-\ell-1\right) d_{k+2} \cdots d_{n},
$$

and from the induction hypothesis we get

$$
\left|\mathcal{Y}_{n-1} \backslash Z_{\mathcal{Y}_{n-1}}(f)\right| \geq\left(d_{k+1}-\left(\ell+d_{1}-1\right)\right) d_{k+2} \cdots d_{n} \geq d_{k+2} \cdots d_{n} .
$$

Adding both inequalities we obtain the desired result.

From now on we can assume that

$$
f \notin I\left(\mathcal{Y}_{n}^{*}\right), f \notin I\left(\mathcal{Y}_{n-1}\right), 0 \leq k<n-1 \text { and } \ell+d_{1}>d_{k+1},
$$

in particular $\ell \geq 1$. In what follows we generalize some methods used by Sørensen [14] to treat projective Reed-Muller codes. Define the set of hyperplanes

$$
\Pi:=\left\{\pi=Z(h) \subset \mathbb{P}^{n}: h=a_{0} X_{0}+\cdots+a_{n-1} X_{n-1}+X_{n} \in K_{n}[X]\right\} .
$$

For all $\pi \in \Pi$, we want to estimate $\left.\mid(\pi \cap \mathcal{X}) \backslash Z_{\mathcal{X}}(f)\right) \mid$.

For each $h=a_{0} X_{0}+\cdots+a_{n-1} X_{n-1}+X_{n}$, define $H: \mathbb{P}^{n} \rightarrow \mathbb{P}^{n}$ by

$$
H\left(x_{0}, \ldots, x_{n}\right)=\left(x_{0}: \cdots: x_{n-1}: h\left(x_{0}, \ldots, x_{n}\right)\right) .
$$

It is easy to see that $H$ is a projectivity that induces a bijection of $\mathcal{X}$ and sends the plane $\pi$ to the plane $Z\left(X_{n}\right)$, in fact

$$
P \in \pi=Z(h) \Longleftrightarrow H(P) \in Z\left(X_{n}\right) .
$$

It is also easy to check that

$$
f(H(X)):=f\left(X_{0}, \ldots, X_{n-1}, a_{0} X_{0}+\cdots+a_{n-1} X_{n-1}+X_{n}\right)
$$


is a homogeneous polynomial of degree $d$, and that the inverse projectivity $H^{-1}$ is the one associated to $h^{*}=-a_{0} X_{0}-\cdots-a_{n-1} X_{n-1}+X_{n}$. Let $g_{h}(X)=f\left(H^{-1}(X)\right)$, then the restriction of the projectivity $H$ to $Z_{\mathcal{X}}(f)$ is a bijection between $Z_{\mathcal{X}}(f)$ and $Z_{\mathcal{X}}\left(g_{h}\right)$ because

$$
P \in Z_{\mathcal{X}}(f) \Longleftrightarrow f(P)=0 \Longleftrightarrow g_{h}(H(P))=0 \Longleftrightarrow H(P) \in Z_{\mathcal{X}}\left(g_{h}\right)
$$

which implies that $H\left((Z(h) \cap \mathcal{X}) \backslash Z_{\mathcal{X}}(f)\right)=\left(Z\left(X_{n}\right) \cap \mathcal{X}\right) \backslash Z_{\mathcal{X}}\left(g_{h}\right)$.

To proceed we consider the following cases, regarding the possibility of $Z_{\mathcal{X}}(f)$ to contain or not a set $\pi \cap \mathcal{X}$, with $\pi \in \Pi$.

(a) Assume that $Z_{\mathcal{X}}(f)$ does not contain any set $\pi \cap \mathcal{X}$, where $\pi \in \Pi$, and define the set of pairs

$$
A_{f}:=\left\{(P, \pi) \in\left(\mathcal{X} \backslash Z_{\mathcal{X}}(f)\right) \times \Pi: P \in \pi\right\} .
$$

Let $\mathcal{X}^{\prime}=\left[K_{0} \times \cdots \times K_{n-1}\right]$ and for every $\pi=Z(h)$ let

$$
g_{h}^{\prime}\left(X_{0}, \ldots, X_{n-1}\right)=g_{h}\left(X_{0}, \ldots, X_{n-1}, 0\right) .
$$

Since $Z(h) \cap \mathcal{X} \not \subset Z_{\mathcal{X}}(f)$ we have that $g_{h}^{\prime}$ does not vanish on $\mathcal{X}^{\prime}$ and is homogeneous of degree $d$. Thus, from $\left|\left(Z\left(X_{n}\right) \cap \mathcal{X}\right) \backslash Z_{\mathcal{X}}\left(g_{h}\right)\right|=\left|\mathcal{X}^{\prime} \backslash Z_{\mathcal{X}^{\prime}}\left(g_{h}^{\prime}\right)\right|$ and the induction hypothesis we get that

$$
\left|(Z(h) \cap \mathcal{X}) \backslash Z_{\mathcal{X}}(f)\right| \geq\left(d_{k+1}-\ell\right) d_{k+2} \cdots d_{n-1} .
$$

So for each $\pi \in \Pi$ we have at least $\left(d_{k+1}-\ell\right) d_{k+2} \cdots d_{n-1}$ points $P$ such that $(P, \pi) \in A_{f}$. From $|\Pi|=d_{n}^{n}$ we have

$$
\left|A_{f}\right| \geq\left(d_{k+1}-\ell\right) d_{k+2} \cdots d_{n-1} d_{n}^{n}
$$

Let $P=\left(b_{0}: \cdots: b_{n}\right) \in \mathcal{X} \backslash Z_{\mathcal{X}}(f)$. If $\left(b_{0}: \cdots: b_{n-1}\right) \neq 0$ then there are $d_{n}^{n-1}$ hyperplanes $\pi \in \Pi$ such that $P \in \pi$. If $P=(0: \cdots: 0: 1)$, there is no hyperplane $\pi \in \Pi$ such that $P \in \pi$, so

$$
\left|A_{f}\right| \leq\left|\mathcal{X} \backslash Z_{\mathcal{X}}(f)\right| d_{n}^{n-1}
$$

From (3.1) and (3.2) we get

$$
\left|\mathcal{X} \backslash Z_{\mathcal{X}}(f)\right| \geq\left(d_{k+1}-\ell\right) d_{k+2} \cdots d_{n} .
$$

(b) Assume that $Z_{\mathcal{X}}(f)$ contains a set $\pi \cap \mathcal{X}$, for some $\pi \in \Pi$. To complete the proof we will consider two subcases.

Subcase b.1: Assume that $d_{k+1}<d_{n}$. Applying the projectivity $H$ corresponding to $\pi$ and passing from $f(X)$ to $f\left(H^{-1}(X)\right)$ we may assume that $\pi=Z\left(X_{n}\right)$. From Lemma 3.6 there exists a homogeneous polynomial $g$ of degree $d-1$ such that $f-g X_{n} \in I(\mathcal{X})$, 
which means $Z_{\mathcal{X}}(f)=Z_{\mathcal{X}}\left(g X_{n}\right)$. For $\tilde{\mathcal{X}}:=\left[1 \times K_{1} \times \cdots \times K_{n-1} \times K_{n}^{\neq 0}\right]$ we have $\mathcal{Y}_{n}^{*} \backslash Z_{\mathcal{Y}_{n}^{*}}(f)=\tilde{\mathcal{X}} \backslash Z_{\widetilde{\mathcal{X}}}(g)$. As before we may get a lower bound for $\tilde{\mathcal{X}} \backslash Z_{\widetilde{\mathcal{X}}}(g)$ by using Theorem 1.6 to obtain a lower bound for the number of nonzero points of $g\left(1, X_{1}, \ldots, X_{n}\right)$ in $K_{1} \times \cdots \times K_{n-1} \times K_{n}^{\neq 0} \in \mathbb{A}^{n}$. To do this we observe that $g\left(1, X_{1}, \ldots, X_{n}\right)$ is a polynomial of degree at most $d-1$, and also that $d_{1} \leq \cdots \leq d_{n-1}$ and $d_{k+1} \leq d_{n}-1$. Thus when we write $K_{1}, \ldots, K_{n-1}, K_{n}^{\neq 0}$ in order of increasing size the set $K_{n}^{\neq 0}$ does not appear before $K_{k+1}$. In [12] the authors prove that this reordering does not affect the lower bound in Theorem 1.6 (2) so we get

$$
\left|\widetilde{\mathcal{X}} \backslash Z_{\widetilde{\mathcal{X}}}(g)\right| \geq\left(d_{k+1}-\ell\right) d_{k+2} \cdots d_{n-1}\left(d_{n}-1\right)
$$

On the set $\mathcal{Y}_{n-1}$ we can use the induction hypothesis, observing that $d-1=\sum_{i=2}^{k+1}\left(d_{i}-1\right)+$ $\ell+d_{1}-d_{k+1}$ and $0<\ell+d_{1}-d_{k+1} \leq d_{k+2}-1$, so

$$
\left|\mathcal{Y}_{n-1} \backslash Z_{\mathcal{Y}_{n-1}}(f)\right| \geq\left(d_{k+2}-\left(\ell+d_{1}-d_{k+1}\right)\right) d_{k+3} \cdots d_{n} \geq\left(d_{k+1}-\ell\right) d_{k+2} \cdots d_{n-1}
$$

Adding both inequalities, we obtain the desired result.

Subcase b.2: Assume that $d_{k+1}=d_{n}$. Let $t \in\{1, \ldots, k+1\}$ be the least index such that $K_{t}=K_{t+1}=\cdots=K_{n}$. For $t \leq j \leq n$ let

$\Pi_{j}=\left\{\pi=Z(h) \subset \mathbb{P}^{n}: h=a_{0} X_{0}+\cdots+a_{j-1} X_{j-1}+X_{j}+a_{j+1} X_{j+1}+\cdots+a_{n} X_{n} \in K_{n}[X]\right\}$.

If for some $j \in\{t, \ldots, n\}$ all sets $\pi \cap \mathcal{X}$, with $\pi \in \Pi_{j}$, are not contained in $Z_{\mathcal{X}}(f)$ then we may use an argument similar to the one used in (a) above to obtain the desired result. In this argument we will use $\Pi_{j}$ instead of $\Pi, \mathcal{X}_{j}^{\prime}=\left[K_{0} \times \cdots \times \widehat{K}_{j} \times \cdots \times K_{n}\right]$ instead of $\mathcal{X}^{\prime}$ (where $K_{0} \times \cdots \times \widehat{K}_{j} \times \cdots \times K_{n}$ means that we omit the set $K_{j}$ in the product) and for every

$$
h=a_{0} X_{0}+\cdots+a_{j-1} X_{j-1}+X_{j}+a_{j+1} X_{j+1}+\cdots+a_{n} X_{n} \in K_{n}[X]
$$

we will set

$$
\begin{aligned}
& g_{h}^{\prime}\left(X_{0}, \ldots, \widehat{X}_{j}, \ldots, X_{n}\right) \\
& =f\left(X_{0}, \ldots, X_{j-1},-a_{0} X_{0}-\cdots-a_{j-1} X_{j-1}-a_{j+1} X_{j+1}-\cdots-a_{n} X_{n}, X_{j+1}, \ldots, X_{n}\right)
\end{aligned}
$$

at the end we use that $\left|\Pi_{j}\right|=d_{n}^{n}=d_{j}^{n}$ to conclude the argument and prove the result. 
If for all $t \leq j \leq n$ there exists $Z\left(h_{j}\right)=\pi_{j} \in \Pi_{j}$ such that $\pi_{j} \cap \mathcal{X} \subset Z_{\mathcal{X}}(f)$ then let $H$ be the projectivity defined by

$$
H\left(x_{0}, \ldots, x_{n}\right)=\left(x_{0}: \cdots: x_{t-1}: h_{t}\left(x_{0}, \ldots, x_{n}\right): x_{t+1}: \cdots: x_{n}\right) .
$$

As before, passing from $f(X)$ to $f\left(H^{-1}(X)\right)$ we may assume that $Z\left(X_{t}\right) \cap \mathcal{X} \subset Z_{\mathcal{X}}(f)$. If all sets $\pi \cap \mathcal{X}$, with $\pi \in \Pi_{t+1}$, are not contained in $Z_{\mathcal{X}}(f)$ then again we may use an argument similar to the one used in (a) above to get the result. If there is some $\pi \in \Pi_{t+1}$ such that $\pi \cap \mathcal{X} \subset Z_{\mathcal{X}}(f)$ then using an appropriate projectivity we may assume that $Z\left(X_{t+1}\right) \cap \mathcal{X} \subset Z_{\mathcal{X}}(f)$ (note that $Z\left(X_{t}\right) \cap \mathcal{X} \subset Z_{\mathcal{X}}(f)$ continues to hold). Proceeding in this manner, we either get the result or we get that $Z\left(X_{j}\right) \cap \mathcal{X} \subset Z_{\mathcal{X}}(f)$ for all $j=t, \ldots, n$, which we assume from now on. From Lemma 3.6, there exists a homogeneous polynomial $g(X)$ of degree $d-(n-t+1)$, such that $f=g \cdot X_{t} \cdots X_{n}$. From $f \notin I\left(\mathcal{Y}_{n}^{*}\right)$ we get that $g$ is not zero on the set $\mathcal{A}=\left[1 \times K_{1} \times \cdots \times K_{t}^{*} \times \cdots \times K_{n}^{*}\right]$ and also that $\left|\mathcal{Y}_{n}^{*} \backslash Z_{\mathcal{Y}_{n}^{*}}(f)\right|=\left|\mathcal{A} \backslash Z_{\mathcal{A}}(g)\right|$. The number of nonzero points of $g$ in $\mathcal{A}$ is the same of the number of nonzero points of $g\left(1, X_{1}, \ldots, X_{n}\right)$ in $K_{1} \times \cdots \times K_{t}^{*} \times \cdots \times K_{n}^{*} \in \mathbb{A}^{n}$. Observe that from the definition of $t$ we get $d_{1} \leq \cdots \leq d_{t-1} \leq d_{t}-1=\cdots=d_{n}-1$ so we may apply Theorem 1.6, noting that $\operatorname{deg}\left(g\left(1, X_{1}, \ldots, X_{n}\right)\right) \leq d-1-(n-t)$. To apply that result we write

$$
d-1-(n-t)=\sum_{i=1}^{t-1}\left(d_{i}-1\right)+\sum_{i=t}^{k}\left(\left(d_{i}-1\right)-1\right)+\ell-(n-k-1)=\sum_{i=1}^{\alpha}\left(\tilde{d}_{i}-1\right)+\tilde{\ell}
$$

where $\tilde{d}_{i}, 0 \leq \alpha \leq k$ and $\tilde{\ell}$ are defined by

$$
\begin{gathered}
\tilde{d}_{i}= \begin{cases}d_{i} & \text { if } 1 \leq i<t, \\
d_{i}-1 & \text { if } t \leq i \leq n,\end{cases} \\
0 \leq \tilde{\ell}=\sum_{i=\alpha+1}^{k}\left(\tilde{d}_{i}-1\right)+\ell-(n-k-1)<\tilde{d}_{\alpha+1}-1
\end{gathered}
$$

(we note that if $t=k+1$ then we omit the term $\sum_{i=t}^{k}\left(\left(d_{i}-1\right)-1\right)$ in (3.4) $)$. With this notation, from Theorem 1.6 we have

$$
\left|\mathcal{A} \backslash Z_{\mathcal{A}}(g)\right| \geq\left(\tilde{d}_{\alpha+1}-\tilde{\ell}\right) \tilde{d}_{\alpha+2} \cdots \tilde{d}_{n} .
$$

Let $a_{\alpha+1}=d_{\alpha+1}-\tilde{d}_{\alpha+1}+\tilde{\ell}$ and $a_{j}=d_{j}-\tilde{d}_{j}$ for $j=\alpha+2, \ldots, n-1$, then

$$
\left(\tilde{d}_{\alpha+1}-\tilde{\ell}\right) \tilde{d}_{\alpha+2} \cdots \tilde{d}_{n-1}=\prod_{i=\alpha+1}^{n-1}\left(d_{i}-a_{i}\right)
$$


and we have

$$
\begin{aligned}
\sum_{i=\alpha+1}^{n-1} a_{i} & =\left(d_{\alpha+1}-\tilde{d}_{\alpha+1}+\tilde{\ell}\right)+\sum_{i=\alpha+2}^{n-1}\left(d_{i}-\tilde{d}_{i}\right)=\tilde{\ell}+\sum_{i=\alpha+1}^{n-1}\left(d_{i}-\tilde{d}_{i}\right) \\
& =\sum_{i=\alpha+1}^{k}\left(\tilde{d}_{i}-1\right)+\ell-(n-k-1)+\sum_{i=\alpha+1}^{k}\left(d_{i}-\tilde{d}_{i}\right)+(n-1-k) \\
& =\sum_{i=\alpha+1}^{k}\left(d_{i}-1\right)+\ell .
\end{aligned}
$$

Thus, from Lemma 1.8 we get $\prod_{i=\alpha+1}^{n-1}\left(d_{i}-a_{i}\right) \geq\left(d_{k+1}-\ell\right) d_{k+2} \cdots d_{n-1}$, and a fortiori

$$
\left|\mathcal{A} \backslash Z_{\mathcal{A}}(g)\right| \geq\left(d_{k+1}-\ell\right) d_{k+2} \cdots d_{n-1}\left(d_{n}-1\right) .
$$

From the induction hypothesis, and similarly as (3.3), we have

$$
\left|\mathcal{Y}_{n-1} \backslash Z_{\mathcal{Y}_{n-1}}(f)\right| \geq\left(d_{k+1}-\ell\right) d_{k+2} \cdots d_{n-1} .
$$

and adding both inequalities we obtain the desired result, which concludes the proof of the Proposition.

We come to the main result of this section.

Theorem 3.8. If $\mathcal{X}$ is the projective nested product of fields over $K_{0}, \ldots, K_{n}$, then the minimum distance of $C_{\mathcal{X}}(d)$ is 1 if $d>\sum_{i=1}^{n}\left(d_{i}-1\right)$. For $1 \leq d \leq \sum_{i=1}^{n}\left(d_{i}-1\right)$, in the case where $d_{1}=\cdots=d_{n}$, or $1 \leq d<d_{r+1}$ in the case where there exists a positive integer $r$ such that $d_{1}=d_{r}<d_{r+1}$, we have

$$
\delta_{\mathcal{X}}(d)=\left(d_{k+1}-\ell\right) d_{k+2} \cdots d_{n}
$$

where $0 \leq k \leq n-1$ and $0 \leq \ell<d_{k+1}-1$ are the unique integers such that $d-1=$ $\sum_{i=1}^{k}\left(d_{i}-1\right)+\ell$

Proof. It is a consequence of Proposition 3.7 and Lemma 3.1 .

As a consequence of our main results we recover the formula for the parameters of Projective Reed-Muller codes.

Corollary 3.9. ([14, Theorem 1]; [13, Proposition 12]) The Projective Reed-Muller code $P C_{d}(n, q)$ is an $\left[\left|\mathbb{P}^{n}\right|, \operatorname{dim} C_{\mathbb{P}^{n}}(d), \delta_{\mathbb{P}^{n}}(d)\right]$-code where

(a) $\left|\mathbb{P}^{n}\right|=\left(q^{n+1}-1\right) /(q-1)$, 
(b) $\operatorname{dim} C_{\mathbb{P}^{n}}(d)=\sum_{j=0}^{n} \sum_{k=0}^{j}(-1)^{k}\left(\begin{array}{l}j \\ k\end{array}\right)\left(\begin{array}{c}j+d-1-k q \\ d-1-k q\end{array}\right)$ and

(c)

$$
\delta_{\mathbb{P}^{n}}(d)=\left\{\begin{array}{cl}
q^{n} & \text { if } \quad 1=d \\
(q-\ell) q^{n-k-1} & \text { if } 1<d \leq n(q-1) \\
1 & \text { if } \quad n(q-1)<d
\end{array}\right.
$$

here $0 \leq k \leq n-1$ and $1 \leq \ell \leq d_{k+1}-1$ are the unique integers such that $d=1+k(q-1)+\ell$.

Proof. From Example 2.3 and Theorems 2.8 and 3.8 we have the result.

Now we present a relationship between the parameters of codes defined over a projective nested product of fields and certain affine cartesian codes.

Corollary 3.10. Let $K_{0}, \ldots, K_{n}$ be subfields of $K$ such that $\mathcal{X}=\left[K_{0} \times K_{1} \times \cdots \times K_{n}\right]$ is a projective nested product of fields and let $\mathcal{X}_{i}^{*}=K_{n+1-i} \times \cdots \times K_{n} \subset \mathbb{A}^{i}$, where $i=1 \ldots, n$. Set $\mathcal{X}_{0}^{*}=\{1\}$ If

$$
C_{\mathcal{X}}(d) \quad \text { is a } \quad\left[|\mathcal{X}|, \operatorname{dim} C_{\mathcal{X}}(d), \delta_{\mathcal{X}}(d)\right] \text {-code }
$$

and

$$
C_{\mathcal{X}_{i}^{*}}(d) \quad \text { is a } \quad\left[\left|\mathcal{X}_{i}^{*}\right|, \operatorname{dim} C_{\mathcal{X}_{i}^{*}}(d), \delta_{\mathcal{X}_{i}^{*}}(d)\right]-\text { code }
$$

then

$$
|\mathcal{X}|=\sum_{i=0}^{n}\left|\mathcal{X}_{i}^{*}\right|, \quad \operatorname{dim} C_{\mathcal{X}}(d)=\sum_{i=0}^{n} \operatorname{dim} C_{\mathcal{X}_{i}^{*}}(d-1) \quad \text { and } \quad \delta_{\mathcal{X}}(d)=\delta_{\mathcal{X}_{n}^{*}}(d-1),
$$

where $\mathcal{X}_{0}^{*}=[1]$ and $\delta_{\mathcal{X}_{n}^{*}}(0):=d_{1} \cdots d_{n}$, with the restriction that if there exists an integer $r$ such that $d_{1}=\cdots=d_{r}<d_{r+1}$, then $d<d_{r+1}$.

Proof. It is a consequence of Theorems 2.8 and 3.8 and [12, Corollary 3.8].

Example 3.11. Let $K=\mathbb{F}_{25}$ be a finite field with 25 elements and let $K_{0}=K_{1}=$ $\mathbb{F}_{5}, K_{2}=\mathbb{F}_{25}$ be subsets of $K$. Then $\mathcal{X}=\left[K_{0} \times K_{1} \times K_{2}\right]$ is a projective nested cartesian product, and the length, the dimension and the minimum distance of the code $C_{\mathcal{X}}(d)$ are:

\begin{tabular}{c|c|c|c|c|c|c|c|c|c|c|c}
$d$ & 1 & 2 & 3 & 4 & 5 & 6 & 7 & 8 & 9 & 10 & 25 \\
\hline$|\mathcal{X}|$ & 151 & 151 & 151 & 151 & 151 & 151 & 151 & 151 & 151 & 151 & 151 \\
\hline $\operatorname{dim} C_{\mathcal{X}}(d)$ & 3 & 6 & 10 & 15 & 21 & 27 & 33 & 39 & 45 & 51 & 141 \\
\hline$\delta_{\mathcal{X}}(d)$ & 125 & 100 & 75 & 50 & 25 & 24 & 23 & 22 & 21 & 20 & 1
\end{tabular}


Observe that for $d=25$, we have $d-1=(5-1)+20$ and for

$$
f=X_{2}\left(X_{2}^{24}-\left(X_{0}^{24}+X_{1}^{24}+2 X_{0}^{4} X_{1}^{20}+2 X_{0}^{20} X_{1}^{4}\right)\right)
$$

we have $w(f)=1<\left(d_{k+1}-\ell\right)=(25-20)=5$.

\section{REFERENCES}

[1] S. Ballet, R. Rolland, On low weight codewords of generalized affine and projective Reed-Muller codes, Des. Codes Cryptogr. 73(2) (2014) 271-297.

[2] B. Buchberger, Ein Algorithmus zum Auffinden der Basiselemente des Restklassenringes nach einem nulldimensionalen Polynomideal. Mathematical Institute, University of Innsbruck, Austria. PhD Thesis. 1965. An English translation appeared in J. Symbolic Comput. 41 (2006) 475-511.

[3] C. Carvalho, On the second Hamming weight of some Reed-Muller type codes, Finite Fields Appl. 24 (2013) 88-94.

[4] C. Carvalho, C., V.G.L. Neumann, Projective Reed-Muller type codes on rational normal scrolls, Finite Fields Appl. 37 (2016) 85-107

[5] A. Couvreur and I. Duursma, Evaluation codes from smooth quadric surfaces and twisted Segre varieties, Des. Codes Cryptogr., 66 (2013) 291-303.

[6] D. Cox, J. Little, D. O'Shea Ideals, varieties and algorithms, Springer-Verlag, 3rd. ed. 2007.

[7] I. Duursma, C. Rentería and H. Tapia-Recillas, Reed-Muller codes on complete intersections, Appl. Algebra Engrg. Comm. Comput., 11 (2001) 455-462.

[8] J. Fitzgerald, R.F. Lax, Decoding affine variety codes using Göbner bases, Des. Codes and Cryptogr. 13(2) (1998) 147-158.

[9] O. Geil, C. Thomsen, Weighted Reed-Muller codes revisited, Des. Codes Cryptogr. 66(1-3) (2013) 195-220.

[10] M. González-Sarabia, C. Rentería and H. Tapia-Recillas, Reed-Muller-type codes over the Segre variety, Finite Fields Appl., 8 (2002) 511-518.

[11] G. Lachaud, The parameters of projective Reed-Muller codes, Discrete Math. 81(2) (1990) 217-221.

[12] H. H. López, C. Rentería-Márquez, R. H. Villarreal, Affine cartesian codes, Des. Codes Cryptogr. 71(1) (2014) 5-19.

[13] C. Rentería, Tapia-Recillas H., Reed-Muller codes: an ideal theory approach, Commu. Algebra 25(2) (1997) 401-413.

[14] A. Sørensen, Projective Reed-Muller codes, IEEE Trans. Inform. Theory 37(6) (1991) 1567-1576. 
Faculdade de Matemática, Universidade Federal de Uberlândia, Av. J. N. Ávila 2121, 38.408-902 - UBERLÂNDIA - MG, BRAZIL

E-mail address: cicero@ufu.br

Faculdade de Matemática, Universidade Federal de Uberlândia, Av. J. N. Ávila 2121, 38.408-902 - UBERLÂNDIA - MG, BRAZIL

E-mail address: victor.neumann@ufu.br

Departamento de Matemáticas, Centro de Investigación y de Estudios Avanzados del iPN, Apartado Postal 14-740, 07000 Mexico City, D.F.

E-mail address: hlopez@math.cinvestav.mx 